\title{
Revista Brasileira de Enfermagem REBEn \\ Erro de medicação: importância da notificação no gerenciamento da segurança do paciente
}

\author{
Medication errors: importance of notification in the management of patient safety \\ Error de medicación: la importancia de la notificación para la seguridad del paciente
}

\section{Elena Bohomol RESUMO}

Enfermeira. Mestre em Enfermagem. Doutoranda em Enfermagem. Docente do Departamento de Enfermagem da UNIFESP,

São Paulo, SP. Membro do Grupo de Estudos e Pesquisa em Administração dos

Serviços de Saúde e Gerenciamento de Enfermagem - UNIFESP ebohomol@denf.epm.br

\section{Laís Helena Ramos}

Enfermeira.

Doutora em Saúde Pública. Professora Adjunto do Departamento de Enfermagem da UNIFESP, São Paulo, SP. Membro do

Grupo de Estudos e Pesquisa em Administração dos Serviços de Saúde e Gerenciamento de Enfermagem - UNIFESP. laisramos@denf.epm.br

Submissão: 13/07/2006 Aprovação: 19/10/2006
A notificação dos erros de medicação é um instrumento importante para o gerenciamento da qualidade da assistência e segurança do paciente. Este estudo objetivou verificar junto à equipe de enfermagem o seu entendimento do que é um erro de medicação e apresentar a sua opinião quanto à notificação deste evento. Foi realizada um survey descritivo/exploratória com 89 profissionais cujos resultados demonstraram uma ausência de uniformidade na compreensão do que é um erro de medicação e quando ele deve ser notificado ao médico ou preenchido o relatório de ocorrências. Concluímos que há necessidade de se desenvolver programas educacionais que elucidem o que são os erros de medicação, discutindo cenários para entender as causas do problema com propostas de melhoria.

Descritores: Erros de medicação; Qualidade; Gerenciamento de segurança; Enfermagem.

\section{ABSTRACT}

Notifying medication errors is an important instrument in managing assistance quality and safety for the patient. The objective of this study was to verify with the nursing team their understanding that a medication error had been committed, and to observe how they notified responsible physicians of the event. To achieve this, researchers used a descriptive/exploratory survey with 89 professionals. The results demonstrate a lack of uniformity in understanding that medication errors had been committed, when these errors must be communicated to a physician, and when an event report must be filled out. The research shows the necessity to develop educational programs that shed light on exactly what constitutes a medication error, clarifying causes of the problem and providing solutions to solve it.

Descriptors: Medication errors; Quality; Safety management; Nursing.

\section{RESUMEN}

La notificación de los errores en la medicación es un instrumiento importante para la gerencia en lo que se refiere a la calidad de la asistencia y seguridad del paciente. Este estudio tiene como objetivo verificar junto al equipo de la enfermaría el entendimiento del mismo sobre lo que es un error de medicación y presentar su opinión referente a la notificación de este evento. Fue realizado un survey descriptivo/ exploratorio con 89 profesionales cuyos resultados demostraron una no-uniformidad en la comprensión de lo que es un error de medicación y cuando este debe ser notificado al medico o notificado en el relatorio de ocurrencias. Concluimos que hay necesidades de sé desenvolver programas educacionales que esclarezcan lo que son errores de medicación, discutiendo escenarios para entender las causas del problema con propuestas de mejoría.

Descriptores: Errores de medicación; Calidad; Administración de la seguridad; Enfermería.

Bohomol E, Ramos LH. Erro de medicação: importância da notificação no gerenciamento da segurança do paciente. Rev Bras Enferm 2007 jan-fev; 60(1):32-6.

\section{INTRODUÇÃO}

O erro, como e por que acontece, seja na área da saúde ou em qualquer outra, abre um leque de possibilidades para estudos relacionados à mente humana e seu aspecto cognitivo, como também, para análise das circunstâncias externas e fatores ambientais.

Os erros podem trazer danos e prejuízos diversos a um paciente, desde o aumento do tempo de permanência em uma instituição hospitalar, necessidade de intervenções diagnósticas e terapêuticas e trazer, até, conseqüências trágicas, como a morte. São conhecidos como eventos iatrogênicos ${ }^{(1)}$.

A palavra iatrogenia é de origem grega e deriva de iatrós que significa médico e gênesis que significa origem ${ }^{(2)}$. Nos primórdios da história da saúde, os eventos iatrogênicos estavam vinculados à atuação médica. No entanto, nos dias de hoje, seu significado tem uma dimensão maior, definida como a "ação 
prejudicial dos profissionais de saúde durante a prestação da assistência" e entendida também, como o "resultado indesejável relacionado à observação, monitorização ou intervenção terapêutica"(3). Devido a eles, a assistência de saúde prestada ao paciente não é tão segura como deveria ser.

O National Center for Health Statistics, nos Estados Unidos da América (EUA), apresentou em 1997, um estudo sobre as razões de morte de pacientes em hospitais americanos e mostrou que 44.000 foram causadas por eventos iatrogênicos. Este montante excedeu as mortes atribuídas a acidentes com carros (43.458), câncer de mama (43.397) eAIDS (16.516). Além dos aspectos relacionados ao indivíduo, foram apresentados os econômicos, ou seja, os custos relacionados às perdas de rendimento profissional, queda de produção doméstica e outros prejuízos correlacionados. Os valores foram estimados entre 17 bilhões e 29 bilhões de dólares, sem considerar as questões jurídicas que normalmente envolvem estas causas ${ }^{(1)}$.

Este tipo de evento tem sido objeto de preocupação dos gestores da saúde, e os erros de medicação, definidos como qualquer erro no processo de prescrição, dispensação ou administração de uma medicação vêm chamando especial atenção, pois são os que mais freqüentemente ocorrem em hospitais, trazem danos ao paciente, contribuem para depreciação profissional e podem, também, aumentar os custos das internações hospitalares ${ }^{(4)}$.

Quando ocorre qualquer dano ou prejuízo relacionado com o uso de uma droga, mesmo que a relação de causa e efeito não possa ser provada, considera-se como evento adverso à medicação $0^{(5)}$. Eles devem ser monitorados e analisados para que medidas preventivas possam ser instauradas, diminuindo assim a possibilidade de novas ocorrências ${ }^{(6)}$.

Há referência de inúmeros métodos usados para sua detecção, que vão desde o relatório individual anônimo, ficha de notificação formal, técnica do incidente crítico, revisão da prescrição, observação direta e combinação do relatório anônimo e observação ${ }^{(6,7)}$. Cada um deles tem suas vantagens e desvantagens, contudo, devem estar adequados aos objetivos das instituições e utilizados como instrumentos gerenciais para a melhoria da qualidade da assistência à saúde.

A Joint Commision on Acreditation of Healthcare Organizations (JCAHO), organização não-governamental americana que avalia a qualidade dos serviços de hospitais dentro dos EUA, recomenda que existam ferramentas para medir e monitorar a performance de uma instituição e propõe a utilização de instrumentos de notificação. Sugere, ainda, que exista uma análise crítica sobre as causas de sua ocorrência e implantação de medidas de qualidade ${ }^{(8)}$.

Sabe-se, no entanto, que o medo e o receio estão presentes quando se aborda uma situação de erro culminando em sub-notificações ou a não notificações dos mesmos, fator preocupante para o gerenciamento de eventos adversos à medicação.

Uma investigação, com enfermeiros, sobre o tema, demonstrou que $40 \%$ de eventos não foram relatados devido ao estigma da atitude negativa em relação ao incidente e os complexos relatórios que os profissionais devem realizar ${ }^{(9)}$.

Outros autores identificaram o medo de represálias e exposição a julgamentos como os principais fatores para a não notificação e referem que apenas $25 \%$ dos erros são formalizados em relatórios ou notificações de ocorrências ${ }^{(10)}$.

A sub-notificação também está vinculada ao desconhecimento do que seja efetivamente um erro de medicação além do desconhecimento frente às intercorrências que possam ocorrer com o paciente e do temor quanto a seu futuro profissional ${ }^{(11,12)}$

Verifica-se que, influenciados pelos programas de acreditação hospitalar brasileiros, muitas instituições hospitalares vêm adotando a notificação de eventos adversos como um indicador para o gerenciamento da qualidade em seus serviços ${ }^{(13)}$.

Recomenda-se, entretanto, que as notificações de erros não sejam dificeis de preencher, não sejam utilizadas como instrumentos para ações disciplinares aos profissionais de saúde e que os dados oriundos destes documentos sejam utilizados para desencadear ações educacionais e mudanças estruturais ou processuais ${ }^{(10,11)}$.

O gerenciamento da qualidade da assistência deve incentivar as ações de participação e valorização dos profissionais, desenvolvendo uma cultura não punitiva dentro das instituições ${ }^{(1,11,12)}$.

\section{OBJETIVOS}

Entendendo que a notificação dos erros de medicação seja um instrumento importante para a melhoria da qualidade da assistência, este estudo tem como objetivo verificar junto à equipe de enfermagem o seu entendimento do que é um erro de medicação e apresentar a sua opinião quanto à notificação do evento e o preenchimento do relatório de ocorrências adversas à medicação.

\section{METODOLOGIA}

O desenho desta pesquisa é não-experimental, na categoria survey descritiva/exploratória. Realizada em uma instituição hospitalar privada na cidade de São Paulo, com 422 leitos, em nove unidades de internação de clínicas médico-cirúrgicas de pacientes adultos. $O$ projeto de pesquisa foi aprovado pela Gerência Executiva de Enfermagem da instituição e pelo Comitê de Ética e Pesquisa da Universidade de afiliação das autoras. A coleta de dados foi realizada ao longo de todo o mês de Fevereiro de 2002.

Esta investigação foi inspirada em um estudo realizado em 1999, em um hospital dos Estados Unidos ${ }^{(10)}$, cujo instrumento de coleta de dados foi cedido pelas autoras norte-americanas com autorização para sua adaptação à realidade brasileira. Foram cumpridas as etapas de tradução para a língua portuguesa; análise da clareza e objetividade do conteúdo por um comitê composto por quatro enfermeiros coordenadores de unidade, quatro enfermeiros educadores e um farmacêutico; análise da estrutura de coleta por profissional estatístico e realização de um teste piloto cujos resultados permitiram os ajustes finais do instrumento de coleta de dados.

A população pesquisada foi a equipe de profissionais do serviço de enfermagem da instituição, composta por 75 enfermeiros, 38 técnicos e 143 auxiliares de enfermagem, totalizando 256 pessoas vinculadas à assistência de enfermagem dos pacientes internados. Foram distribuídos aleatoriamente 124 questionários, dos quais retornaram 89 que vieram com o Consentimento Livre e Esclarecido devidamente assinado, perfazendo uma amostra de $34,7 \%$.

As informações contempladas no instrumento de coleta de dados foram divididas em três partes: caracterização sócio-demográfica da amostra; apresentação de cinco cenários contendo situações que podem ser vivenciados pelos profissionais de enfermagem no seu cotidiano; e, cinco perguntas relacionadas ao preenchimento do relatório de ocorrências para os erros de medicação.

Para a análise estatística optou-se pelo o teste de Qui-quadrado com nível de significância de $5 \%$ e utilizado o software SPSS $®$.

\section{RESULTADOS E DISCUSSÃO}

A amostra foi constituída de 49 (55\%) de auxiliares de enfermagem, 13 (15\%) técnicos de enfermagem e $27(30 \%)$ de enfermeiros. O perfil sóciodemográfico obtido foi: 54 (60,7\%) do sexo feminino; 40 (44,9\%) com idade entre 30 e 39 anos; $33(37,1 \%)$ com tempo de formado entre 6 e 10 anos; $42(47,7 \%)$ com tempo de trabalho na instituição entre 0 e 2 anos. A amostra identificou que $50(56,8 \%)$ dos profissionais trabalhavam apenas na instituição pesquisada sem outro vinculo empregatício ou educacional. Foram representados os períodos de trabalho em: manhã $(35,2 \%)$, tarde $(34,1 \%)$ e noturno $(30.7 \%)$. 


\section{Avaliação dos Cenários}

Baseado nos cinco diferentes cenários apresentados para a equipe de enfermagem, a Tabela 1 apresenta as respostas dos mesmos, com alternativas sim ou não, se houve um erro de medicação, se a situação deveria ou não ser notificada para o médico responsável e se haveria necessidade de realizar o preenchimento de um relatório sobre erros de medicação.

Os resultados revelam que em quatro Cenários $(2,3,4$ e 5$)$ os sujeitos acreditam tratar-se de um erro de medicação. No entanto, no Cenário 1 , quando existe uma omissão na administração de uma medicação, a maioria (79,0\% sim) não caracteriza a ocorrência como um erro. A omissão é um erro de medicação definida como a não administração de uma dose prescrita para o paciente ${ }^{(14)}$.

Cabe ressaltar que neste cenário o paciente encontrava-se em outro setor e, provavelmente, a equipe entendeu que a responsabilidade de sua administração fosse dos profissionais daquele serviço. Situações como estas são encontradas na literatura explicando ser esta uma das causas para a não administração de medicação, porém tais fatos não devem ser rotineiros e sugere-se que políticas institucionais devam ser implantadas para que o paciente não tenha sua terapêutica prejudicada ${ }^{(15)}$.

O Cenário 2 apresenta uma situação relacionada ao atraso, que é um erro de horário, isto é, administração de medicação fora do intervalo de tempo predefinido no prontuário do paciente ${ }^{(14)}$. As circunstâncias que levam ao atraso de uma medicação são inúmeras, que vão desde a falta da medicação na unidade, um grande número de itens para serem administrados, muitos pacientes internados, demora na dispensação das medicações, até um dimensionamento de pessoal inadequado. Contudo, elas devem ser analisadas e, se possivel, bloqueadas, pois tais situações podem ser compreendidas em função de um dia incomum, ou serem repetitivas, devido a problemas no processo de trabalho dentro da instituição ${ }^{(16)}$.

O Cenário 3 trata da programação da uma bomba de infusão, cuja velocidade do gotejamento estava incorreta, proporcionando uma dose maior do que a prescrita. Autilização de tecnologia para o apoio à assistência é sempre bem vinda, contudo, se a mesma não for utilizada adequadamente pode contribuir para o aparecimento de ocorrências adversas. Para prevenir tais situações, há necessidade de manter os profissionais capacitados e treinados em sua manipulação, bem como estabelecer programas de controle e manutenção preventiva de equipamentos ${ }^{(16)}$.

Tabela 1. Respostas da equipe de enfermagem aos cinco cenários relacionados a administração de medicação. São Paulo, 2002.

\begin{tabular}{|c|c|c|}
\hline Item & $\frac{\operatorname{Sim}}{\mathrm{n}^{\circ}(\%)}$ & $\begin{array}{c}\text { Não } \\
\mathrm{n}^{0}(\%)\end{array}$ \\
\hline \multicolumn{3}{|c|}{$\begin{array}{l}\text { Cenário 1: } A \text { um paciente não é administrada sua dose oral de ampicilina do meio-dia porque } \\
\text { ele estava em um exame de raios } X \text { durante } 3 \text { horas }\end{array}$} \\
\hline $\begin{array}{l}\text { Erro de medicação } \\
\text { Notificação ao médico } \\
\text { Preenchimento do relatório }\end{array}$ & $\begin{array}{l}16(21,0) \\
35(46,6) \\
38(50,0)\end{array}$ & $\begin{array}{l}60(79,0) \\
40(53,4) \\
38(50,0)\end{array}$ \\
\hline \multicolumn{3}{|c|}{$\begin{array}{l}\text { Cenário 2: Em uma clinica cirúrgica bastante movimentada, quatro pacientes receberam as } \\
\text { suas doses intravenosas de antibiótico das } 8 \text { da manhã, com } 4 \text { horas de atraso. }\end{array}$} \\
\hline $\begin{array}{l}\text { Erro de medicação } \\
\text { Notificação ao médico } \\
\text { Preenchimento do relatório }\end{array}$ & $\begin{array}{l}60(76,9) \\
54(71,0) \\
74(93,6)\end{array}$ & $\begin{array}{r}18(23,1) \\
22(29,0) \\
5(6,4) \\
\end{array}$ \\
\hline \multicolumn{3}{|c|}{$\begin{array}{l}\text { Cenário 3: Um paciente está recebendo nutrição parenteral total. A bomba de infusão foi } \\
\text { programa para } 200 \mathrm{ml} / \mathrm{h} \text { em } 24 \text { horas ao invés de } 125 \mathrm{ml} / \mathrm{h} \text { em } 24 \text { horas, com inicio às } 4 \text { horas } \\
\text { da manhã. Na troca do plantão, portanto às } 7 \text { horas da manhã, um membro da equipe de } \\
\text { enfermagem programou a bomba para o gotejamento correto. }\end{array}$} \\
\hline $\begin{array}{l}\text { Erro de medicação } \\
\text { Notificação ao médico } \\
\text { Preenchimento do relatório }\end{array}$ & $\begin{array}{l}78(94,0) \\
51(68,0) \\
73(92,4)\end{array}$ & $\begin{array}{r}5(6,0) \\
24(32,0) \\
6(7,6)\end{array}$ \\
\hline \multicolumn{3}{|c|}{$\begin{array}{l}\text { Cenário 4: Um paciente foi admitido em sua unidade com mal asmático às } 4 \text { horas da manhã } \\
\text { do dia 25/12/01, com prescrição de inalação a cada } 4 \text { horas. A enfermagem não deu a dose } \\
\text { das } 4 \text { da tarde deste dia de Natal porque o paciente estava dormindo. }\end{array}$} \\
\hline $\begin{array}{l}\text { Erro de medicação } \\
\text { Notificação ao médico } \\
\text { Preenchimento do relatório }\end{array}$ & $\begin{array}{l}43(54,4) \\
25(33,8) \\
41(54,0)\end{array}$ & $\begin{array}{l}36(45,6) \\
49(66,2) \\
35(46,0)\end{array}$ \\
\hline \multicolumn{3}{|c|}{$\begin{array}{l}\text { Cenário 5: Um médico prescreveu Tylex } 1 \text { comprimido, para caso de dor no pós-operatório. } \\
\text { Às } 4 \text { da tarde, o paciente queixou-se de dor requisitando o medicamento e foi medicado. Às } \\
\text { 6:30 hs da tarde, um segundo comprimido foi solicitado pelo paciente e foi dado pelo } \\
\text { funcionário responsável. }\end{array}$} \\
\hline $\begin{array}{l}\text { Erro de medicação } \\
\text { Notificação ao médico } \\
\text { Preenchimento do relatório }\end{array}$ & $\begin{array}{l}72(86,7) \\
66(84,6) \\
66(83,5)\end{array}$ & $\begin{array}{l}11(13,3) \\
12(15,4) \\
13(16,5)\end{array}$ \\
\hline
\end{tabular}

$n<89$ devido a respostas em branco

Tabela 2. Respostas da equipe de enfermagem quanto ao preenchimento do relatório de ocorrências para Erros de Medicação. São Paulo, 2002.

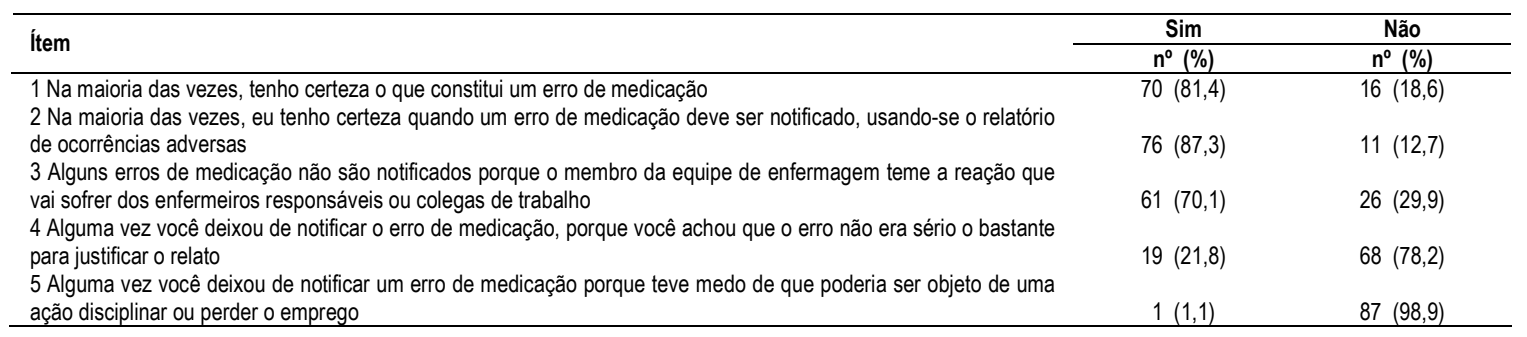


Em relação ao Cenário 4, os sujeitos responderam tratar-se de um erro de medicação, uma vez que a inalação não foi administrada. Em contrapartida, a literatura apresenta circunstâncias semelhantes ${ }^{(10,17)}$, em que a maioria dos profissionais avaliou que o sono do paciente era um indicador de que não havia mais desconforto respiratório e que ele receberia sua medicação ao despertar, sem prejuízo do tratamento. Tal circunstância abre espaço para uma discussão sobre a autonomia dos profissionais de enfermagem e limites de sua ação quanto à manutenção ou suspensão de uma medicação.

O Cenário 5 enfoca a administração de uma segunda dose de opióide codeína, depressor do sistema nervoso central em um curto espaço de tempo, por solicitação do paciente. Os profissionais pesquisados referiram tratar-se de um erro, inferindo-se que, a autorização para uma nova administração devesse ser autorizada pelo médico. Cabe ao profissional médico, legalmente, a prescrição de medicamentos aos pacientes, realizando a indicação do uso, bem como a dose, freqüência, via, bem como as instruções necessárias para a sua administração.

Estes achados se contrapõe às investigações publicadas ${ }^{(10,17)}$, em que profissionais de enfermagem referiram não caracterizar-se um erro, uma vez que o paciente tinha dor e conseqüentemente nova dose poderia ser administrada. Explicam tratar-se de uma decisão que deve ser baseada na experiência e na competência do pensamento crítico do enfermeiro.

A Tabela 1 apresenta, ainda, se as situações encontradas nos cenários deveriam ser notificadas ao médico responsável e se um relatório deveria ser preenchido. Os resultados revelam que, nos Cenários 2, 3 e 5, em que os profissionais entenderam ser um erro, eles tendem a notificar ao médico e preencher o relatório. Contudo, no Cenário 4, mesmo que as pessoas considerem a não realização da inalação um erro, sua notificação ao médico não deveria ocorrer.

Há, também, uma polarização de quase $50 \%$ a $50 \%$ nas respostas dos cenários 1 e 4 quanto ao preenchimento de um relatório o que sugere, uma falta de uniformidade de conduta e entendimentos de como agir em tais situações.

\section{Relatório de Notificação de Erros de Medicação}

A Tabela 2 apresenta as respostas a cinco perguntas relacionadas ao preenchimento do relatório de ocorrências, cujas alternativas eram sim ou não.

A maior parte dos sujeitos pesquisados $(81,4 \% \mathrm{sim})$ referiu saber o que constitui um erro de medicação, quando o relatório de ocorrências deveria ser preenchido ( $87,3 \%$ sim) e que as razões para a não notificação dos erros estão relacionadas ao temor da "reação que vai sofrer dos enfermeiros responsáveis ou colegas de trabalho" (70,1\% sim). Entretanto, a maioria dos profissionais não deixou de relatar o evento por achar que o erro não era sério o bastante $(78,2 \%$ não) e, também, que não temem as ações disciplinares ou perdas de emprego que poderiam ocorrer devido à ocorrência de um erro ( $98,9 \%$ não).

Estas respostas chamam atenção para a necessidade de clarificar para a equipe de enfermagem os conceitos do que é um erro de medicação e os tipos de erros que existem. Isto, porque, há um paradoxo entre a "certeza do que se constitui um erro de medicação" ( $81,4 \%$ sim , $18,6 \%$ não) e a concordância apresentada nos cenários, em especial no Cenário 1 (21\% sim, 79,0\% não) e Cenário 4 ( $54,4 \%$ sim, $45,6 \%$ não), mesmo tratandose de um erro.

Esta pesquisa identifica um gap entre a percepção do conhecimento da equipe de enfermagem sobre o tema e seu real conhecimento, abrindo possibilidades para que as instituições desenhem programas educacionais para promover este reconhecimento e orientações específicas de como gerenciar os erros ${ }^{(4,10,12,16,17)}$.

Este estudo, também, lança luzes quanto à forma de gerenciar os erros em uma instituição de saúde. A administração não punitiva encontra apoio na literatura ${ }^{(1,11,16)}$ encorajando os profissionais a relataram os erros e acidentes potenciais para que os processos de trabalho sejam revistos $e$ tragam uma assistência segura ao paciente.

\section{CONCLUSÃO}

Dos cinco cenários que representam situações de erros de medicação, verificou-se que a equipe julgou tratar-se de um erro de medicação em quatro; que os mesmos deveriam ser notificados ao médico em três e que o relatório de ocorrências deveria ser preenchido em quatro, destacandose, contudo, que houve polarização da opinião da equipe em 50\% a $50 \%$ no cenário em que os mesmos julgaram não se tratar de um erro.

Na opinião da maioria dos profissionais, os mesmos têm certeza do que se constitui um erro de medicação $(81,4 \%)$, quando o mesmo deve ser notificado ( $87,3 \%)$, não temem realizar a notificação por medo de uma ação disciplinar (98,9\%) e acreditam que muitos erros não são notificados pelos membros da equipe de enfermagem, pois este profissional teme a reação que vai sofrer dos enfermeiros responsáveis e colegas de trabalho $(70,1 \%)$.

No grupo pesquisado não houve uma uniformidade na compreensão do que é um erro de medicação, quando deve ser notificado ao médico e preenchido o relatório de ocorrências.

\section{CONSIDERAÇÕES FINAIS}

O erro de medicação é uma questão multiprofissional e as circunstâncias que o envolvem são multifatoriais, não se limitando apenas a uma categoria profissional.

A equipe de enfermagem é sensivel ao problema pois está envolvida no sistema de medicação e muitas vezes é responsabilizada pelos erros, temendo aos julgamentos e reações que podem ocorrer.

$O$ conhecimento adquirido com esta pesquisa reforça a necessidade de se desenvolver programas educacionais que elucidem o que são os erros de medicação, discutindo cenários para entender as causas do problema e propostas de melhoria.

A administração dos serviços de saúde deve estar voltada a desenvolver um sistema de trabalho para reduzir ou eliminar as barreiras para a notificação dos erros de medicação, focando a segurança do paciente como um padrão de alta qualidade da assistência à saúde.

\section{REFERÊNCIAS}

1. Kohn LT, Corrigan JM, Donaldson MS, editors. To err is human building a safer health system. Committee on quality of health care in America, Institute of Medicine. Washington (DC): National Academy Press; 2000.

2. Cunha AG. Dicionário etimológico Nova Fronteira da língua portuguesa. Rio de Janeiro (RJ): Nova Fronteira; 1982.

3. Padilha KG. Ocorrências iatrogênicas na prática de enfermagem em Unidades de Terapia Intensiva (tese livre-docência). São Paulo (SP): Escola de Enfermagem, Universidade de São Paulo; 1998.
4. Cassiani SHB. Administração de medicamentos. São Paulo: EPU; 2000.

5. Van den Bet PMLA, Egberts TCG, de Jong-van den Berg LTW, Brouwers JRBJ. Drug-related problems in hospitalized patients. Drug Saf 2000;22(4):321-33.

6. Flynn EA, Barker KN. Medication Errors Research. In: Cohen MR. Medication Errors. Causes, prevention adn risk management. Boston (MS): Jones and Bartlett Publishers; 1999.

7. Flynn EA, Barker KN, Pepper GA, Bates DW, Mikeal R. Comparison of methods for detecting medication errors in 36 
hospitals and skilled-nursing facilities. Am J Health-Syst Pharm 2002; 59(1):436-45.

8. Schneider PJ, Gift M. Measuring and monitoring the performance of the medication use system. In: Cousins DD. Medication Use: A Systems approach to reducing errors. Oakbrook Terrace (IL): Joint Commission on Accreditation of Healthcare Organizations: 1998. p.19-37

9. Day G. Improving medication administration through an enhanced occurrence reporting system. J Nurs Care Qual 1994;9(1):51-6.

10. Osborne J, Blais K, Hayes JS. Nurses's perceptions: when is it a medication error? J Nurs Adm 1999;29(4):33-8

11. Carvalho VT, Cassiani SHB. Análise dos comportamentos dos profissionais de enfermagem frente aos erros na administração de medicamentos. Acta Paul Enferm 2002;15(2):45-54.

12. Bohomol E. Erros de Medicação: causas e fatores desencadeantes sob a ótica da equipe de enfermagem. (dissertação). São Paulo (SP): Universidade Federal de São Paulo; 2002.
13. ONA - Organização Nacional de Acreditação. Manual das organizações prestadoras de serviços hospitalares. Coleção Manuais de Acreditação. Brasília (DF): Organização Nacional de Acreditação; 2004.

14. Cousins DM. Defining medication errors. In: Cousins DM. Medication use: a systems approach to reducing errors. Oakbrook Terrace (IL): Joint Commission on Accreditation of Healthcare Organizations; 1998. p.39-56.

15. Baker HM. Rules outside the rules for administration of medication: a study in New South Wales, Australia. J Nurs Scholarsh 1997;29(2):155-8.

16. Smetzer J. Take 10 giant steps to medication safety. CE Connection. Nursing; 2001. (cited 2002 Mai 05). Available from: URL: http://www.springnet.com

17. Mayo AM, Duncan D. Nurse perceptions of medication errors. What we need to know for patient safety. J Nurs Care Quality 2004;19(3):209-217. 